\title{
Sao Tome and Principe
}

\section{STRUCTURE OF HIGHER EDUCATION SYSTEM}

\section{Description}

Higher education is the responsibilty of the Ministerio da Educação, Cultura e Ciência and is still in its early stages. Higher education is currently provided by one public institution and a few private institutions. There exists three university-level degrees, and although the law does provide for doctorates, none of the institutions currently offers doctoral programmes of their own.

\section{Stages of studies}

\section{University level first stage}

\section{Undergraduate}

The first stage of university-level education is made up of the Bacharel, awarded after three years' study, and the Licenciatura, awarded after four years' study, or one year's study following the Bacharel

\section{University level second stage}

\section{Graduate level}

The Mestrado is awarded after two years' study following the Licenciatura

\section{ADMISSION TO HIGHER EDUCATION}

\section{Admission to university-level studies}

Name of Secondary school credential required: Certificado de Fin de Estudos Secundarios

\section{NATIONAL BODIES}

\section{Ministério da Educação, Cultura e Ciência (Ministry of Education, Culture and Science)}

Minister: Olinto da Silva Daio

Rua Samora Machel, Agua Grande CP 41
São Tomé

Tel: +239222 3366

Website: http://www.mecc.gov.st

Data for academic year: 2016-2017

Source: IAU from UNESCO/BREDA Sao Tomé-et-Principe: rapport d'état du système éducatif, 2017.

\section{Institution}

\section{University of São Tomé and Príncipe (USTP)}

Rua da Caixa

São Tomé

Tel: +239(2) 22-44-23

Website: http://www.ustp.st

Rector: Aires Bruzaca

\section{Course/Programme}

Agronomy (Agronomy); Economics (Economics); Education (Education); Engineering (Computer Engineering; Engineering; Information Technology; Telecommunications Engineering); Health Sciences (Anaesthesiology; Health Administration; Health Sciences; Nursing); History (History); Law (Law); Modern Languages (French; Portuguese); Natural Sciences (Biology; Geography; Mathematics; Natural Sciences; Physics); Public Relations and Communication (Communication Studies; Public Relations)

History: Founded as "Higher Polytechnic Institute of São Tomé and Príncipe" (ISPSTP) in 1996, acquired present title and status 2014.

Accrediting agency: Ministério da Educação, Cultura e Ciência

Degrees and diplomas: Bacharelato / Bacharel (Education; Nursing), Licenciatura (Agronomy; Arts and Humanities; Economics; Engineering; Health Sciences; Law; Management; Modern Languages; Natural Sciences)

Last Update: $31-08-2017$ 